\title{
Classes sociales, classes scolaires : une polémique sur l'éducation en 1819-1820
}

\section{Yvan Lamonde}

Volume 41, 1974

URI : https://id.erudit.org/iderudit/1007241ar

DOI : https://doi.org/10.7202/1007241ar

Aller au sommaire du numéro

Éditeur(s)

Les Éditions Historia Ecclesiæ Catholicæ Canadensis Inc.

ISSN

0318-6172 (imprimé)

1927-7067 (numérique)

Découvrir la revue

Citer cet article

Lamonde, Y. (1974). Classes sociales, classes scolaires : une polémique sur l'éducation en 1819-1820. Sessions d'étude - Société canadienne d'histoire de l'Église catholique, 41, 43-59. https://doi.org/10.7202/1007241ar

Tous droits réservés @ Les Éditions Historia Ecclesiæ Catholicæ Canadensis Inc., 1975
Ce document est protégé par la loi sur le droit d'auteur. L'utilisation des services d'Érudit (y compris la reproduction) est assujettie à sa politique d'utilisation que vous pouvez consulter en ligne.

https://apropos.erudit.org/fr/usagers/politique-dutilisation/ 


\section{Classes sociales, classes scolaires : une polémique sur l'éducation en 1819-1820}

Une analyse des structures des occupations en rapport avec l'ethnicité à Québec et à Montréal (1819-1844) incitait Fernand Ouellet à voir dans les disparités de niveaux d'instruction l'origine des disparités socio-économiques entre les groupes ethniques francophone et anglophone. Pour M. Ouellet, cette disparité dans les niveaux d'instruction résultait de la divergence des intérêts politiques investis dans l'éducation même ${ }^{1}$.

La présente analyse d'une polémique sur l'éducation en 18191820 corrobore l'interprétation de $\mathrm{M}$. Ouellet tout en dégageant les positions prises, à l'occasion de cette polémique, par les groupes sociaux intéressés au contrôle de l'éducation. Cette disparité de savoir reliée à une disparité de pouvoir tient à une neutralisation des efforts en raison des divergences irréconciliables des intérêts et à une emprise cléricale progressive sur l'éducation. Enfin cette polémique, soulevée par un prêtre "français 》, l'abbé de Calonne, éclairera le rôle joué par ce clergé français contre-révolutionnaire et permettra de mieux situer l'introduction de Lamennais au Québec ${ }^{2}$.

\section{A l'origine de la polémique : l'abbé de Calonne (1743-1822)}

De famille noble, «chassé » par la Révolution française, établi depuis 1807 à Trois-Rivières à titre de chapelain des Ursulines, l'abbé de Calonne se retrouvait dans une "petite France " (pourtour du lac St-Pierre) partiellement desservie entre 1795 et 1841 par dix prêtres français réfugiés au Bas-Canada après 1789. Cette recrue de 47 prêtres français royalistes entre 1791 et 1800 devait marquer quantitativement et qualitativement une Église catholique bas-cana-

1 «Structures des occupations et ethnicité dans les villes de Québec et de Montréal (1819-1844) 》, Eléments d'histoire sociale du Bas-Canada, Montréal, HMH (Cahiers du Québec), 1972, pp. 177-202; «L'enseignement primaire : responsabilité des Églises ou de l'État (1801-1836) 》, Ibidem, pp. 259277.

2 T. Matheson a déjà brièvement abordé ce dernier sujet dans « La Mennais et l'éducation au Bas-Canada ", Revue d'Histoire de l'Amérique française, 13, 4 (mars 1960): 476-491. 
dienne dont la situation précaire tenait au manque d'effectifs et à l'incertitude de son statut légal ${ }^{3}$.

"Éprouvé », l'abbé de Calonne n'était pas homme à cacher ses couleurs. Trois ans après son arrivée à Trois-Rivières, la crise politique de 1810 , qui reposait à nouveau le problème de l'aménagement des rapports entre l'Église, l'État et la Chambre d'Assemblée, allait faire connaître ses positions et ses relations. "Appelé au Conseil » avec $\mathbf{M}^{\mathrm{gr}}$ Plessis «comme pouvant seconder les vues du Gouvernement et servir au maintien du bon ordre dans la Province ${ }^{4}$ ", l'abbé de Calonne partageait les craintes du gouverneur Craig :

Je suis bien revenu de l'idée que le gouverneur avait trop pris l'alarme. En vérité, Monseigneur, c'est plus violent qu'on ne pense communément. Il a raison de dire que ce sont tous les principes français. On a répandu des écrits dans ma paroisse, et ce n'est rien, mais on en a endoctriné quelques-uns qui sont complètement pervertis. Il y en a un entre autres qui a tenu les propos les plus incendiaires jusqu'à parler de révolte et d'aller en force enlever ceux qui sont arrêtés si on ne les faisait sortir de prison.

Si on lui parle des massacres de France : - Il n'y aurait pas de mal qu'il y en eut ici.

Si on lui oppose les autres malheurs, il répond qu'il n'y a pas de peuple plus heureux aujourd'hui que les Français; si on lui oppose ce que j'ai dit en chaire : - Je le crois bien, dit-il, ces prêtres français sont tous des rebelles qui se sont enfuis pour échapper à la punition.

Cela serait très risible, si cela ne conduisait pas ces malheureux à leur perte. Mais vous voyez comme on les endoctrine, car le pauvre homme n'est pas capable de l'imaginer quoique très capable de le propager. Heureusement que le nombre est infiniment petit. Mais que ne fait-on pas accroire à des gens simples, on avait bien persuadé au peuple parisien que les aristocrates avaient fait miner la Seine pour la faire sauter au besoin. Ce que je vois à la Pointe-du-Lac me fait croire qu'on emploie des moyens sourds et très dangereux

3 Jean-Pierre WaLlot, «La religion catholique et les Canadiens au début du XIX siècle», Un Québec qui bougeait, Montréal, Éd. du Boréal Express (coll. 17/60), 1973, pp. 183-224; Claude GALARNEAU, La France devant l'opinion canadienne (1760-1815), Québec, PUL (Cahiers de l'Institut d'Histoire, \# 16), 1970, pp. 211-224.

$4 \mathrm{Mgr}$ Plessis à M. Burke, vicaire général, Halifax, 16 août 1810. Archives de l'archevêché de Québec [AAQ], RL, 7: 190-191. 
pour pervertir le peuple. Nous sommes bien heureux d'avoir un gouverneur très ferme et très vigilant.

J'ai tenté un instant d'écrire au gouverneur pour lui faire voir qu'il serait très politique de laisser venir ici une douzaine de prêtres français dont les principes sont si bien connus et si bien éprouvés 5 .

Victime «des principes français», l'abbé, visé en plus comme "prêtre français » fuyard, était à même de les reconnaître et de les dénoncer avec «véhémence 6 》, tout comme les «moyens 》 pris pour les «propager », y compris l'éducation. Son loyalisme, tout comme celui de ses concitoyens curés de la «petite France», dont l'abbé Jean Raimbault de Nicolet ${ }^{7}$, n'avait pas fait défaut :

J'ai fait le service paroissial dimanche dernier, j'en ai profité pour parler pendant près d'une heure sur la proclamation /du gouverneur/... 8 .

L'abbé de Calonne continua d'être informé principalement des " effets désastreux 》 de ces «principes français 》 par une abondante correspondance reçue de sa famille, d'amis et de son agent d'affaires 9 en France, et par la lecture de journaux, dont l'Ambigu, feuille antibonapartiste publiée à Londres, et l'Ami de la Religion et du Roi, dirigé par M. Picot, confrère des abbés Raimbault et Fournier (Baiedu-Febvre) au Séminaire d'Orléans ${ }^{10}$. Ses correspondants, tout

5 Abbé de Calonne à Mgr Plessis, [c. 15 avril 1810], lettre citée dans Histoire du Monastère des Ursulines des Trois-Rivières, Trois-Rivières, P.V. Ayotte, 1892, Tome II, pp. 100-101.

6 M. Raimbault à Mgr Plessis, 8 avril 1810, 13 nov. 1814, 18 déc. 1815, Archives du Séminaire de Nicolet [ASN]. Lettres de M. Raimbault à Mgr Plessis et à Mgr Panet (1806-1831); Mgr Plessis à Raimbault, ASN, [c. 8 fév. 1816]. Lettres de Mgr Plessis à Raimbault.

7 M. Raimbault à Mgr Plessis, 5 avril 1810. ASN, loc. cit., ou, AAQ, $\mathrm{SN}, \mathbf{a - 4 1 .}$

8 Abbé de Calonne à [Mor Plessis ?], [fin mars - début avril 1810], Histoire du Monastère..., op. cit., II, p. 102.

9 Archives du Collège de Sainte-Anne-de-la-Pocatière [ACSAP], de Calonne 36 et 37 (1795-1822). Les «Lettres adressées à M. de Calonne» (registre) aux Archives des Ursulines de Trois-Rivières sont le plus souvent des transcriptions des originaux aux ACSAP.

10 M. Raimbault à Mgr Plessis, ASN, 7 sept. 1816, 29 août 1817, loc. cit.; Mgr Plessis à M. Raimbault, ASN, 12, 26 sept., 1er oct. 1816, 21 juin, 3 sept., 30 déc. 1817, 31 janv., $1^{\text {er }}$ mars 1819, 2 nov. 1820, loc. cit.; M. Raimbault à Mgr Plessis, Archives de l'évêché de Nicolet [AEN], 20 nov. 1818, 11 et 14 fév. 1819; Mgr Plessis à M. Roux, 3 nov. 1817, AAQ, RL, 9: 252; M. de St-Quentin, Paris, à l'abbé de Calonne, 10 juillet 1818, ACSAP, Calonne 36, LX; même au même, 13 avril 1819, ACSAP, Calonne 37, V. 
comme ceux de l'abbé Raimbault, par exemple, le tenaient au courant, à deux mois près, des nouvelles «voix» de l'Église qui commençaient à se faire entendre en France :

... qu'à la faute irréparable de la révolution française se rattachent des fautes plus graves encore! que la tolérance avec tous ses abus est moins capable d'arrêter les écarts de la raison humaine que la simple voix de l'apôtre de l'Évangile à laquelle il est si doux de se rendre et qui semble néanmoins cette voix qui crie dans le désert.

Celle de l'abbé Fraissinous tonne à St-Sulpice, deux à trois mille hommes de tous les âges, de toutes les conditions composent l'auditoire toutes les fois qu'il prononce un discours. Confesseur éloquent de la religion, contre lincrédulité, il entraîne les uns par le brillant de son débit, terrasse les autres par la force de ses argumens, commande l'intérêt à la plupart et imprime le respect à tous. Malgré le danger de s'essayer après lui dans ce genre, de jeunes Lévites marchent à pas de géant sur ses traces, ils sont l'ornement de l'autel, ils seront un jour l'orgueuil de la tribune apostolique 11.

À cette restauration de la prédication à la «tribune » catholique correspondait un renouveau de la littérature, auquel la bibliothèque de 800 volumes de l'abbé de Calonne indiquait la familiarité; il connaissait Fleury, les Pères de l'Église, Bourdaloue, de Bonald et Lamennais ${ }^{12}$ :

il paraît depuis un an un ouvrage intitulé sur l'indifférence de la religion, par l'abbé de Lamenais. il est à sa 4 e édition. Cet ouvrage est sans contredit le plus beau de ce siècle. je vais dire à Mr. de St-Quentin de vous l'envoyer ${ }^{13}$.

Un mois plus tard, son agent d'affaires écrivait :

Je vous envoie aussi un livre extrêmement intéressant que j'ai acheté à la recommandation de votre neveu. C'est un Essai sur l'indifférence en matière de religion, on dit que c'est un

11 M. Durier, Paris, à l'abbé Raimbault, 21 mars 1819, ASN, Séminaire III, 57.

12 Rév. G. Carron à l'abbé de Calonne, 2 janv. 1812. ACSAP, Calonne 36, XXIII; D. de St-Quentin, Paris, au même, 28 août 1817, loc. cit., XIII; même au même, 10 juillet 1818, loc. cit., LX; Histoire du Monastère..., op. cit., t. II, pp. 55-56, 92, 127.

13 M. D'Aubers, Paris, à l'abbé de Calonne, 16 mars 1819, ACSAP, Calonne 37, IV. 
chef-d'œuvre. il est comme vous voyez à sa quatrième Édition à 4,000 volumes chacune ${ }^{14}$.

Ce renouveau du prosélytisme entraîna l'abbé de Calonne, loyaliste véhément, à contrer ici les efforts de diffusion des "principes français », l'Essai de Lamennais s'avérant d'ailleurs adaptable à " l'indifférence » religieuse ${ }^{15}$ au Bas-Canada, telle que notée par l'épiscopat et le clergé et attribuable à la marque imprimée à l'Église par trois événements : l'établissement de l'Église anglicane après 1763; la montée sociale, grâce au parlementarisme, des gens. de professions libérales; et surtout le manque crucial d'effectifs cléricaux.

\section{La polémique de 1819-1820 sur l'éducation}

L'empressement du curé français de la Baie-du-Febvre, CharlesVincent Fournier, à surveiller l'établissement d'une école dans sa paroisse relevait de la même vigilance, affinée par l'expérience de la Révolution que le zèle de l'abbé français Jean Raimbault au Séminaire de Nicolet et celui du prestigieux abbé de Calonne à seconder, en 1810, les vues du Gouvernement :

... l'orage commence déjà à se former, on crie déjà au fanatisme. Dans la Chambre d'Assemblée, des Canadiens ont montré des principes impies, ils ne voulaient rien moins qu'enlever aux prêtres l'éducation de la jeunesse, et aux religieuses l'administration des malades.

... dans le Parlement, qui se tient tous les ans à Québec, à l'instar de celui de l'Angleterre, des membres décidèrent de placer, aux frais du Gouvernement, des « écoles à la Lancastre ». Comme je ne suis pas lancastrien et que je ne suis pas du tout libéral, mes idées n'étant point par conséquent libérales, je viens de donner la chasse à un homme libéral, qui était venu ici pour montrer d'une manière libérale; j'ai acheté l'emplacement qu'il venait de louer, et, tout de suite, j'en ai acheté un autre proche de l'église, où il y a maintenant une école de garçons ${ }^{16}$.

14 D. de St-Quentin à l'abbé de Calonne, 13 avril 1819, ACSAP, Calonne 37, V.

15 Mgr Plessis au Rév. Raby, 28 janv. 1811, AAQ, RL, 7: 251; M. Ciquard, curé franççais de Saint-François-du-Lac, à $\mathbf{M g r}$ Plessis, 5 mai 1813, AEN, Saint-François-du-Lac, no 9; abbé Raimbault, curé français de Nicolet, à Mgr Plessis, 5 mars 1816, AEN.

16 Abbé C.-V. Fournier à Madame de Loynes de Morett, 20 juillet 1817, citée dans Joseph-Elzéar Bellemare, Histoire de la Baie-St-Antoine dite Baie-du-Febvre, Montréal, Impr. La Patrie, 1911, pp. 186-196. 
Non seulement l'Église n'avait-elle pas appuyé la loi de 1801, sanctionnée par l'État pour l'établissement d'écoles primaires, mais elle voyait comme dangereuse l'initiative de la Chambre d'Assemblée, donc de laïcs de professions libérales, de promouvoir en 1815 l'établissement d'écoles d'État empruntant au britannique Joseph Lancaster sa méthode d'enseignement. Les écoles «lancastriennes 》, ouvertes aux enfants, indifféremment de leur appartenance religieuse, utilisaient les élèves les plus avancés comme «moniteurs 》, de façon à généraliser l'instruction à des coûts minima ${ }^{17}$. Des "libéraux 》 proposaient donc des écoles lancastriennes «libérales », avec des maîtres « libéraux 》.

Ces écoles «royales» à méthode lancastrienne, dont l'une était établie à Trois-Rivières et dirigée par un maitre anglais, connurent précisément en 1819 le premier sommet de leur croissance numérique.

Fort de son loyalisme, de son expérience, de son prestige, de sa vigilance et de ses lectures, l'abbé de Calonne, faisant pour la première fois mention publique du nom de Lamennais, transmettait à la Gazette des Trois-Rivières les réflexions suivantes :

\section{Mr le Rédacteur,}

Je vois que des Sociétés de Londres, montrent beaucoup de zèle, et se donnent beaucoup de mouvemens, pour procurer de l'éducation aux jeunes canadiens. Ce zèle qui se porte si loin, a-t-il pour but de nous donner des principes et des mœurs? j'avoue que je n'eus jamais pensé que deux articles si essentiels, nous fussent venus de Londres. Quelques-uns de ceux qui nous ont été envoyés pour conduire cette entreprise, m'ayant fait l'honneur de me faire part de leur projet, je leur fis franchement quelques objections, auxquelles ils répondirent aussi franchement, que dans leurs écoles on ne parloit point de Religion.

Ayez la bonté, Monsieur, d'insérer dans votre feuille les brièves réflexions suivantes. Elles sont tirées d'un ouvrage très récent et très célèbre en France. J'ose dire que Bossuet s'en seroit fait honneur. Ce livre est intitulé : De l'indifférence en matière de Religion. Avant que le second volume paroisse, le

17 Louis-Philippe Audet, Histoire de l'enseignement au Québec, Montréal, Holt, Rinehart et Winston, 1971, t. I, pp. 254-271, 357, 366-368. Un «sommaire d'un plan pour instruire deux mille enfants», de Joseph Lancaster, était paru dans la Gazette de Québec du 4 août 1808. 
premier a été réimprimé quatre fois en un an, au nombre de douze mille exemplaires chaque fois.

Voici les réflexions de cet auteur, page 500 :

- Ne l'oublions jamais, la religion est l'unique éducation du peuple. Sans la Religion il ne sauroit rien, rien sur tout de ce qu'il importe le plus à la société qu'il sache, et à lui de savoir. Il ignoreroit également et les devoirs de l'homme et sa destinée. Il végéteroit au milieu des Académies, des Universités, des Gymnases, dans un abrutissement, cent fois pire que l'état sauvage. La Religion le civilise; elle nourrit le pauvre de vérités comme elle le nourrit de pain; elle éclaire, elle agrandit son intelligence; et le dernier des petits enfans instruits à son école, plus véritablement philosophe qu'aucun des prétendus sages qui ne reconnoissent d'autre guide que leur raison, confondroit, le Catéchisme à la main, cette raison altière, par la sublimité de ses enseignemens. Il étoit digne d'un philosophe matérialiste, en substituant les évolutions * à des instructions, et en mettant entre les mains une pierre muette, en place du livre où il puisoit ces hautes et importantes leçons, \&c. »

J'ai l'honneur d'être, Monsieur,

Votre très-humble \&c.

DE CALONNE, Prêtre.

* L'auteur parle ici des Écoles Lancastriennes, qui, malgré les cris et les efforts de nos soi-disans philosophes, n'ont pu s'introduire, presque nulle part en France. Il en sera de même ici. Elles crouleront avant d'avoir pu se glisser dans aucune de nos campagnes ${ }^{18}$.

Ce nouveau prosélytisme clérical s'exprimant dans les «papiers publics » allait entraîner une polémique diffusée dans six journaux du Bas-Canada: La Gazette des Trois-Rivières, la Gazette de Québec, le Canadien de Québec, la Gazette de Montréal, le Spectateur canadien de Montréal et le Courrier du Bas-Canada de Montréal. Critiqué par divers interlocuteurs, l'abbé de Calonne publia une seconde lettre à la rédaction, plus nuancée, dans la Gazette des Trois-Rivières :

18 Gazette des Trois-Rivières (GTR), 9 nov. 1819; reproduit dans Gazette de Québec (GQ), 15 nov.; Le Canadien, 17 nov.; Spectateur Canadien (SC), 20 nov.; Gazette de Montréal (GM), 1er déc.; le Courrier du BasCanada $(C B C)$ ne semble pas avoir reproduit le texte. 
Je ne suis ni vénérable, ni prélat : je suis vieux, et mon éducation a eu pour base la religion, qui dans les tristes evenemens d'une vie trop agitée, m'a été d'un grand soutien et une grande consolation.

la lettre que vous avez eu la bonté d'insérer dans votre feuille, se réduit à cette seule proposition : il ne peut $\mathrm{y}$ avoir de bonne éducation, si la religion n'en est la base; ou plus brièvement, la religion est la base d'une bonne éducation. Il n'y a ni bon protestant, ni bon catholique, ni homme grave, qui puisse contredire cette vérité. Aussi $\mathbf{Y} Z$ n'a-t-il plus même effleuré la question : et dans tous ces entortillemens, il n'a pas osé énoncer la contradictoire. La proposition est restée intacte, parce qu'elle est inattaquable. Les mots d'Y $Z$ et de toute la cohorte de l'alphabet, ne l'ébranleront pas.

J'ai plaisanté sur les écoles à la Lancastre ou à la Madras, parce qu'il est très plaisant que notre siècle de lumières se persuade que nous avons été dix-huit siècles sans un bon plan d'éducation, et que celui qui a formé les Newton, les Leibnitz, les Euler, les Bossuet, les Fénelon, les Mallebranche, tous personnages religieux, doive le céder aux méthodes à la Lancastre ou à la Madras.

Vous êtes trop bon d'avoir supprimé ce qui vous a paru trop mordant. Je ne crains pas plus les traits piquants d'Y Z, que la force de ses arguties.

J'ai l'honneur d'être, Monsieur,

Avec reconnaissance,

Votre très humble, \&c.

DE CALONNE ${ }^{19}$.

Les répliques vinrent rapidement : de l'anglophone et rédacteurpropriétaire de la Gazette de Québec, John Neilson, de cinq correspondants anonymes «libéraux » et d'un membre du clergé, l'abbé Charles-François Painchaud de Ste-Anne-de-la-Pocatière, qui signait «Campagnard ». De novembre 1819 à février 1820, cette discussion allait en quelque sorte rendre publics les intérêts des «partis» concernés par le contrôle de l'éducation; l'offensive des « libéraux » contre l'abbé de Calonne justifia l'entrée en scène de l'abbé Painchaud.

19 GTR, 30 nov. 1819; SC, 4 déc.; GQ, 6 déc.; GM, 8 déc.; Canadien, 8 déc. 
Répliquant en anglais et en français, John Neilson allait droit au but en montrant l'insuffisance du «seul catéchisme»:

Quelque grands et quelque nombreux qu'aient été les maux produits par une fausse philosophie et par l'indifférence religieuse, ils ne sont pas, selon nous comparables aux maux qui ont été la suite de l'ignorance générale...

À ceux qui concevaient l'instruction comme « ornement de l'esprit », Neilson écrivait :

Ils pourraient revenir ces jours heureux où le peuple s'achetait et se vendait avec le sol, où il était taillable et corvéable à merci et miséricorde, où l'esprit était serf aussi bien que le corps...

L'instruction, nécessaire "pour réussir dans les affaires de la vie », devait d'ailleurs être particulièrement favorisée au Bas-Canada :

Que personne ne suscite des obstacles à l'éducation générale, mais plutôt que tous aient compassion d'un peuple qui se trouve tous les jours obligé d'entrer en concurrence avec des étrangers dans le commerce et les affaires de la vie, sans avoir eu les mêmes avantages...

Et répondant ponctuellement au «second Bossuet 》 de l'abbé de Calonne, Neilson rappelait :

... et qu'ils n'oublient jamais que sans peuple, il n'y aurait point de religion.

Enfin, Neilson précisait à l'abbé de Calonne qu'il aurait dû dès le départ se borner à son second texte, à sa seconde proposition, à savoir que la religion est la «base » de l'éducation, «quoiqu'il fut inutile de mettre en proposition sur les Gazettes ce que personne ne nie $20 \gg$ !

Tout comme Neilson, un correspondant anonyme mettait en évidence la nécessité de l'instruction dans un contexte de révolution économique et technologique :

Si l'on disait que c'est un mal [simplifier l'enseignement grâce à la méthode de Lancaster], autant vaudrait dire qu'il faut brûler tous les moulins à eaux ou à vapeur et nous remettre

$20 G Q, 18$ nov. 1819; 22 nov., 16 déc.; reproduit dans Canadien, 24 nov. 1819; SC, 27 nov.; GTR, 30 nov.; GM, 1er déc. 
à moudre le bled à bras, comme on le faisait avant la renaissance des arts... ${ }^{21}$

La bourgeoisie libérale avait des raisons un peu différentes de celles de la bourgeoisie commerciale de promouvoir l'éducation. Des Canadiens, au libéralisme issu des Lumières et de la Révolution et soucieux de la «diffusion » de ces lumières par l'éducation, ne manquèrent pas de qualifier l'abbé de Calonne «d'apologiste de l'ignorance » et de faire voir la source du «mal , dans l'ignorance :

Le premier écrit de $M$. de Calonne et ceux auxquels il a donné lieu ne font pas peu de bruit dans quelques-unes de nos paroisses. Les personnes instruites blâment généralement le vénérable abbé d'avoir, sans doute contre son opinion, confirmé les cultivateurs de ce pays dans une indifférence et une apathie en fait d'éducation, dont il était déjà bien difficile de le tirer. Les ignorants, au contraire, et surtout ceux d'entre eux que l'avarice domine, n'ont pas appris sans une secrète satisfaction ce que paraissait penser un membre de notre respectable clergé. Plusieurs d'entre ces derniers n'ont pu s'empêcher, m'a-t-on dit, de manifester au dehors cette joie insensée en annonçant qu'ils allaient retirer leurs enfants des écoles, ou qu'ils renonceraient au dessein qu'ils avaient eu de les y envoyer 22 .

Ces laïcs que «l'improbation » de l'abbé de Calonne n'inquiétait pas prirent d'ailleurs plaisir à l'invectiver en le traitant de «pédant », en qualifiant «d'incorrectes 》 les réflexions de son «célèbre auteur $^{23}$ 》 et en faisant état de la confusion de certaines discussions :

... ce n'était pas mon intention d'entrer en discussion sur un sujet qui ne peut conduire qu'à un labyrinthe, d'où on ne sort guère plus avancé qu'on était, lorsqu'on y est entré, je veux dire celui de la religion ${ }^{24}$.

Le démocratisme de ces libéraux trouva sa meilleure expression dans les deux interventions de celui qui signait $A B C$. Refusant d'être «dupe de la perfide hypocrisie » de l'abbé qui, faute d'avoir de

21 « De la boîte. Un mot sur l'écrit de M. de Calonne», $C B C, 27$ nov. 1819.

22 Philomathès, "Pour le spectateur canadien 》, SC, 15 janv. 1820.

$23 \mathrm{YZ}$, «Mr. Le Rédacteur», GTR, 23 nov. 1819; Canadien, 1er déc.; ANTI-Pas, «Communication》, GM, 24 nov.

$24 \mathrm{YZ}$, «À Philalethès », GTR, 21 déc. 1819; Philalethès publia dans GTR, 7 déc. 1819 et 25 janv. 1820. 
bonnes raisons de s'opposer à l'instruction du peuple, devait * payer d'effronterie », $A B C$ rappelait qu'en France, depuis le retour des Bourbons, l'instruction des basses classes était devenue « une affaire et un intérêt de parti» et que «les mêmes motifs qui leur [royalistes] font désirer le retour à l'ancien ordre de choses les portent aussi à s'opposer au progrès de l'instruction »; " ceux qui s'engraissaient de la substance du peuple et dont les plaisirs étaient dus en partie à sa misère, avaient donc intérêt qu'il fût ignorant jusqu'à l'aveuglement », comme en témoignait encore l'état d'ignorance, donc de dépravation de certains pays européens comme l'Espagne, l'Italie, l'Irlande ${ }^{25}$. Répliquant à la seconde lettre de l'abbé de Calonne, ABC précisait que les écoles de Madras et de Lancaster ne visaient pas «à former des Newton 》, mais « à apprendre aux enfants du peuple à lire, à écrire et à compter, et cela, avec le moins de difficulté, de tems et de dépenses possibles ${ }^{26} \gg$.

L'intervention de "Campagnard, de Ste-Anne-de-la-Pocatière », pseudonyme de l'abbé Charles-François Painchaud, curé et fondateur du Collège de Sainte-Anne, complétait l'éventail des groupes d'intérêt représentés jusqu'alors dans la polémique. Après un prêtre "français », un bourgeois anglophone et quelques « libéraux », un prêtre canadien exprimait la position de l'Église.

Painchaud - alias Campagnard - désavouait ${ }^{27}$ le texte de Lamennais cité par l'abbé de Calonne, «signalé comme français et catholique 28 , expliquant que «ce qui peut être vrai et bon dans un pays peut ne l'être pas dans un autre 29 »; pour Painchaud, de Calonne avait publié un second texte plus nuancé pour «se retirer de l'embarras ${ }^{30} \gg$. Il reprochait au noble prêtre français son attitude élitiste, rappelant qu'il était «urgent que les Canadiens sachent lire et écrire eu égard à leur position politique » concurrentielle avec les Britanniques et les Américains :

Car dans l'ordre social, quelle est la cause de la différence parmi les hommes (...)? N'est-ce pas l'éducation? Si l'éducation seule peut opérer une si grande différence parmi les

$25 \mathrm{ABC}$, «Pour le Spectateur Canadien》, SC, 4 et 11 déc. 1819 et 29 janv. 1820.

$26 \mathrm{ABC}$, «Pour le Spectateur Canadien 》, SC, 11 déc. 1819.

$27 G Q, 2$ mars 1820; Canadien, 8 mars. Lettre datée du 26 fév. 1820.

$28 G Q, 16$ déc. 1819; Canadien et SC, 25 déc.

$29 G Q, 2$ mars 1820; Canadien, 8 mars. Lettre datée du 26 fév. 1820.

30 Canadien, 22 déc. 1819, 19 et 26 janv. 1820; lettre du 29 nov. 1819. 
hommes, pourquoi donc mettre une barrière insurmontable à ce pauvre homme du peuple pour parvenir à ce que la société appelle les biens et les honneurs? Mr. l'abbé et tous ceux qui pensent comme lui (et il y en a un grand nombre) voudront sans doute conserver ces avantages à une certaine classe d'hommes privilégiés de la société et faire grâce d'enseigner au peuple le catéchisme ${ }^{31}$.

Ce souci «d'ordre social» s'alimentait chez l'abbé Painchaud à l'urgence pour le peuple de pouvoir participer à la vie politique (gazettes, législature, question des subsides) et d'atteindre aux «biens » et aux « honneurs».

En désaccord partiellement avec l'abbé de Calonne, l'abbé Painchaud dénonçait agressivement les libéraux qui, dans une "Chambre d'Assemblée en grande partie composée de Catholiques », avaient «consenti à la passation d'un projet de loi pour l'établissement d'écoles gratuites dont la corporation est nommée par le Gouverneur ${ }^{32}$ ». L'abbé Painchaud répliquait à $\mathrm{ABC}^{33}$ 《 qui a paru, sous cette armature philosophique et libérale, un de ces adeptes dangereux, qui, comme le serpent, ne présentent d'abord que la tête à l'ouverture, mais qui ont bientôt fait passer tout leur corps si on ne les arrête à temps ${ }^{34}$ »; selon le prêtre-éducateur, «ce n'est nullement pour nous donner des mœurs que Mess. les libéraux se tourmentent tant (...). On connaît l'arbre aux fruits. Voyez ce qu'ils ont fait en France sous un autre nom. Ils s'efforçaient d'instruire le peuple; mais dans quelles vues ? Ce n'était que pour frayer une route, dans le cour de la nation, au pouvoir destructeur de leur fausse et pernicieuse philosophie, et par là, renverser, comme ils l'ont fait, le trône et l'autel ${ }^{35}$ ». Ce faisant, ils renversaient « l'ordre social »:

Ces messieurs n'ignorent pas que dans toutes les classes il y a conjuration contre l'autorité, que l'inférieur profite de tous les moyens de s'élever si non au-dessus, au moins au niveau de son supérieur; c'est la marche ordinaire de l'orgueuil si naturel à l'homme, et surtout à l'homme irréligieux ${ }^{36}$.

31 lbidem.

32 lbidem.

33 Réplique de $\mathrm{ABC}, S C, 29$ janv. 1820.

$34 G Q, 2$ mars 1820; Canadien, 8 mars. Lettre datée du 26 fév. 1820.

35 « $\mathrm{A}$ John Neilson», GQ, 13 janv. 1820; SC, 22 janv.; Canadien, 26 janv. Lettre du 24 déc. 1819.

36 Ibidem. 
Au contraire, «la doctrine » de l'Église en éducation, c'est d'élever les enfants «dans la crainte du Seigneur et l'obéissance au Roi ${ }^{37}$ ».

Finalement, l'abbé Painchaud, cinq ans avant la loi des Écoles de Fabrique (1824), préconisait ce système :

Le parlement a fait des efforts répétés pour donner aux paroisses la surintendance sur leurs écoles. Il n'a pas encore réussi. Qu'il s'y prenne donc d'une autre manière, en donnant annuellement l'argent aux principaux de chaque paroisse pour soutenir des écoles 38 .

Peut-être même les «Écoles des frères de la doctrine chrétienne » pourraient-elles contribuer à la propagation des lumières religieuses, qui seules peuvent assurer le rétablissement de l'ordre ${ }^{39}$ »? Au-delà d'une certaine forme récupératrice de promotion de l'instruction, l'abbé Painchaud prenait position pour un conservatisme social d'Ancien Régime :

... mais quel but se propose-t-on dans cette diffusion des lumières répandues si indistinctement parmi les bons comme parmi les méchants? Qu'attend-on de ces armes si dangereuses entre les mains de ceux qui ne savent et ne veulent que détruire ? Je suppose charitablement que c'est le bonheur de la masse du peuple. Or j'en atteste ici tous les siècles passés sans oublier le présent, j'en atteste tous les chantres du bonheur de la vie, et je demande si de tous tems l'ignorance des champs n'a pas été le séjour du bonheur, plutôt que la science et les beaux arts des cités? Les poètes n'en ont jamais chanté d'autres; c'est cette vérité incontestable qui a fait soutenir si victorieusement à J.J. Rousseau que les arts et les sciences ont plus nui que servi au bonheur des hommes 40 .

\section{Mosaïque de tensions et de distorsions}

En faisant ressortir la polyvalence des intérêts placés dans l'éducation, cette polémique de trois mois dans les «papiers publics» permet d'identifier les "partis » en cause et de dégager des composantes des forces sociales investies dans l'éducation. Dans quelle mesure était-on conscient des besoins d'instruction du peuple et quels étaient les obstacles à la réalisation de ces besoins ?

$37 G Q, 16$ déc. 1819; Canadien et $S C, 25$ déc.

$38 G Q, 2$ mars 1820; Canadien, 8 mars. Lettre datée du 26 fév. 1820.

$39 G Q, 16$ déc. 1819; Canadien et $S C, 25$ déc.

40 Canadien, 22 déc. 1819, 19 et 26 janv. 1820; lettre du 29 nov. 1819. 
La croissance de l'immigration anglophone après 1815 et la révolution économique au Bas-Canada avaient certes rendu impérieuse l'instruction élémentaire des Canadiens français, en situation évidente de concurrence. Et Neilson et l'abbé Painchaud s'entendaient sur ce besoin nouveau venu avec les exigences de la production agricole, du commerce et de l'administration publique. Les marchands avaient besoin de producteurs et de producteurs qualifiés.

Les gens de professions libérales, qui, précisément en 1820 , atteignaient un seuil maximal d'effectifs ${ }^{41}$, croyaient, en progressistes, à l'instruction, contre l'ignorance et l'obscurantisme; la valorisation de l'instruction n'était-elle pas une conséquence logique des « Lumières »? Mais ces «libéraux 》 canadiens-français favorisaient, contre le clergé, un système d'écoles laïc, neutre, et contre l'Exécutif du Gouvernement opposé à la responsabilité de la Chambre d'Assemblée, un système d'écoles public, certes, mais relevant de la Chambre d'Assemblée, c'est-à-dire de leur pouvoir et de leur prétention nationale à la représentation populaire.

L'alarme cléricale des prêtres français et d'un de Calonne provoqua le clergé «canadien » à réagir à un problème - l'éducation élémentaire - dont ces « éprouvés » connaissaient l'importance idéologique. D'abord dirigés contre les libéraux et leur « vénin », qui avaient renversé l'ordre en renversant le trône et l'autel, les écrits de l'abbé Painchaud proposaient un système d'écoles producteur de politiciens, d'une élite; c'était bien un fondateur de séminaire-collège qui écrivait ainsi. Souhaitée un moment par Painchaud, l'égalité en éducation était récusée en raison de «l'orgueil 》 des « inférieurs 》 à vouloir s'élever, et en raison, finalement, des vertus de «l'ignorance des champs ».

Il y avait donc, en 1819, conscience des besoins d'éducation. Mais de quelle éducation? Celle de quel "parti », de quel pouvoir? Un système scolaire pouvait-il en même temps être neutre et catholique, relever de la paroisse, de l'Exécutif, de la Chambre d'Assemblée ? Un maître d'école pouvait-il être protestant, anglophone, laïc, lancastrien...?

\section{Vers le contrôle clérical sur l'éducation (1820-1840)}

Les écoles à «système " lancastrien et de Madras continuèrent de s'établir; leur popularité persistait grâce aux efforts des libéraux :

41 F. OUellet, «Structure des occupations... 》, loc. cit., pp. 180-186. 
L'enseignement mutuel triomphe de toutes les résistances de la routine et de toutes les menées sourdes des amis des ténèbres. (...) Lancaster est selon eux un homme qu'on aurait dû étouffer comme Voltaire. L'imposture a besoin de l'ignorance ${ }^{42}$.

Selon un « vieux chrétien», « tout système [était] bon pourvu qu'il ait pour principe l'instruction mutuelle ${ }^{43}$ ». Le protonotaire JosephFrançois Perrault publiait en 1821 un Cours d'éducation élémentaire à l'usage de l'école gratuite... carrément favorable à la méthode lancastrienne d'enseignement mutuel. Les évêques surveillaient :

Le mal ne peut qu'augmenter depuis que le gouvernement s'est déclaré patron de cette école lancastrienne [celle de Perreault]; il ne sera pas aisé d'en arrêter le progrès 44 .

La loi des écoles de Fabrique (1824) ne fut pas un succès, compte tenu de l'indifférence du clergé en matière d'éducation élémentaire et de la grande importance accordée, au plan de l'administration paroissiale, aux lieux et aux objets du culte ${ }^{45}$. Les libéraux combattaient toujours :

Nos philosophes québécois ont résolu paraît-il de détruire le principe du Bill d'éducation passé l'an dernier pour introduire dans la Chambre d'Assemblée le système biblique gazé sous le nom de Lancaster. Le protonotaire Perrault qui en est enjoué ne s'aperçoit pas que les Borgia et Co. veulent s'en servir pour ruiner l'influence du clergé, qui de son côté n'a pas mis assez de zèle à faire ériger dans les campagnes les écoles en vertu du dernier Bill. Il faut faire avorter ce nouveau plan d'impiété 46.

L'évêque de Québec répondit :

Beaucoup de curés se remuent pour avoir des écoles sur le principe du Bill de l'année dernière; mais il faut du temps pour les établir. Prenons patience. Je n'ai pas encore vu le

42 «Éducation (Lancaster) 》, GM, 12 janv. 1820. Extrait du Journal du Commerce de Paris du 24 août 1819.

43 «De l'éducation... », Le Canadien, 31 mai 1820.

$44 \mathrm{Mgr}$ Lartigue à Mgr Plessis, 21 oct. 1823, Archives de la Chancellerie de l'Archevêché de Montréal [ACAM], RLL, 2: 266.

45 Richard СНАВот, Le curé de campagne et la contestation locale au Québec (de 1792 aux troubles de 1837-38), Montréal, HMH (à paraître): chap. I; Voir la polémique autour de Lancaster dans Le Canadien, oct. 1824 fév. 1825.

46 Mgr Lartigue à Mgr Plessis, 27 janv. 1825, ACAM, RLL, 3: 159. 
Bill des Écoles Lancastriennes et ne sais pas ce que l'on prétend en faire. Croyez que les membres de l'Assemblée ne sont pas aisés à mener. Je leur trouve un peu trop de libéralité en matière de religion... 47

Passée par les libéraux, l'importante loi des Écoles d'Assemblée (1829), qui allait multiplier les effectifs scolaires, ne devait pas être reconduite en 1836. Liée à un parti qui se radicalisait politiquement de plus en plus, cette législation scolaire importait trop à un clergé - et à l'évêque Lartigue du nouveau diocèse de Montréal - pour qu'il n'y ait pas affrontement d'intérêt et de pouvoir :

Outre qu'elle [une démarche pour des Écoles de Fabrique contre des écoles d'Assemblée] ferait honneur à l'Église c'est le seul moyen d'arracher la génération future à une éducation détestable; et je voyais dernièrement par les journaux que les laïcs renouvelleraient le plus tôt possible leur mauvaise loi de 1829 , si nous négligions cette occasion précieuse, et peutêtre unique, de nous emparer de l'instruction de la jeunesse 48.

Le clergé allait accaparer l'enseignement primaire, les « Frères de la Doctrine chrétienne » en tête, multiplier les collèges "classiques » et ouvrir la première université. Les niveaux de pouvoir scolaire s'emboîtaient... mutuellement.

\section{Conclusions}

Cette polémique de 1819 , soulevée par un prêtre français, indique le rôle de vigilance doctrinale joué par ce clergé contrerévolutionnaire établi au Bas-Canada. Alerté, ce clergé français pouvait reconnaître les dangers et sonner l'alarme, comme l'atteste ce premier prosélytisme journalistique.

L'abbé de Calonne inaugurait, à l'occasion de cette polémique, la tradition menaisienne au Bas-Canada en recourant à un Lamennais conservateur, ultramontain, que l'abbé Lartigue allait bientôt découvrir ${ }^{49}$. C'était d'ailleurs à propos de cette question «mi-temporelle, mi-spirituelle» que pouvait être, l'éducation, selon l'Église, qu'un prêtre monarchiste donnait une première cohérence, une

47 Mgr Plessis à Mgr Lartigue, 2 fév. 1825, AAQ, RL, 12: 179-180.

$48 \mathrm{Mgr}^{\mathrm{gr}}$ Lartigue à Mgr Signay, 1er $^{\mathrm{er}}$ ai 1836, AAQ, DM, G: 52.

49 François BeAudin, «L'influence de La Mennais sur Mgr Lartigue, premier évêque de Montréal», Revue d'Histoire de l'Amérique française, 25, 2 (sept. 1871): 225-237. 
première cible à une tradition ultramontaine qui trouva ici dans l'éducation son terrain de confusion le plus fertile.

Ce débat autour de l'école "lancastrienne » et «libérale », autour du pouvoir de contrôle sur l'éducation, permet de voir clairement les forces sociales en action dans cette question et de comprendre pourquoi il ne pouvait y avoir de résultante efficace de ces composantes orientées de façons trop divergentes. L'absence de résultante - ou la résultante cléricale - explique à sa façon la disparité des niveaux d'instruction, la disparité socio-économique des groupes ethniques.

Enfin, cet échec de l'école primaire libérale ne signifiait point l'échec de la méthode d' "enseignement mutuel », qui fut d'ailleurs adoptée après 1840 par le clergé au niveau des écoles primaires. En effet, lorsque des réformistes, puis des libéraux plus radicaux, se soucièrent après 1840 d'instruction «populaire » en fondant des Instituts Canadiens en vue de «l'instruction mutuelle " et par des moyens $d$ " «entraide mutuelle », ils relançaient une tradition libérale qui prétendait à l'hégémonie de l'éducation. Mais par stratégie, les moyens avaient changé... Et ceci est une autre histoire!

Yvan LAMONDE, Centre d'Etudes canadiennes-françaises, Université McGill. 